\title{
中国北方温带灌丛生态系统碳、氮、磷储量
}

\author{
郭炎培 ${ }^{1}$ 杨 弦 ${ }^{1}$ 安尼瓦尔.买买提 ${ }^{2}$ 刘鸿噟 $^{1}$ 马文红 ${ }^{3}$ 于顺利 ${ }^{4}$ 唐志尧 $1^{*}$ \\ ${ }^{1}$ 北京大学城市与环境学院, 北京 $100871 ;{ }^{2}$ 中国科学院新疆生态与地理研究所, 乌鲁木齐 $830011 ;{ }^{3}$ 内蒙古大学生命科学学院, 呼和浩特 $010021 ;{ }^{4}$ 中 \\ 国科学院植物研究所植被与环境变化国家重点实验室, 北京 100093
}

摘 要 研究生态系统碳 $(\mathrm{C}) 、$ 氮 $(\mathrm{N})$ 、磷 $(\mathrm{P})$ 密度分布和储量对于理解生态系统碳循环和养分循环的机制和规律有重要意义。 现有的相关研究多集中在森林和草地生态系统。在中国北方, 灌从生境水分和土壤条件差异很大, 这为研究生态系统C、 $\mathrm{N} 、$ $\mathrm{P}$ 密度与储量的分布格局提供了良好条件。该研究调查了 433 个中国北方温带灌从样地的生物量、调落物以及土壤等组分的 有机C及 $\mathrm{N} 、 \mathrm{P}$ 含量, 据此计算出中国北方灌从生态系统有机C及 $\mathrm{N} 、 \mathrm{P}$ 密度和储量。结果表明: 中国北方灌从平均生态系统有 机C及 $\mathrm{N} 、 \mathrm{P}$ 密度分别为 $69.8 \mathrm{Mg} \cdot \mathrm{hm}^{-2} 、 7.3 \mathrm{Mg} \cdot \mathrm{hm}^{-2} 、 4.2 \mathrm{Mg} \cdot \mathrm{hm}^{-2}$ 。其中, 生物量C、N、P密度分别为 $5.1 \mathrm{Mg} \cdot \mathrm{hm}{ }^{-2} 、 11.5 \times 10^{-2}$ $\mathrm{Mg} \cdot \mathrm{hm}^{-2} 、 8.6 \times 10^{-3} \mathrm{Mg} \cdot \mathrm{hm}^{-2}$, 生物量 $\mathrm{C} 、 \mathrm{~N} 、 \mathrm{P}$ 密度与降水和土壤养分关系显著; 调落物 $\mathrm{C} 、 \mathrm{~N} 、 \mathrm{P}$ 密度分别为 $1.4 \mathrm{Mg} \cdot \mathrm{hm} \mathrm{m}^{-2} 、 3.8 \times 10^{-2}$ $\mathrm{Mg} \cdot \mathrm{hm}^{-2} 、 2.5 \times 10^{-3} \mathrm{Mg} \cdot \mathrm{hm}^{-2}$, 调落物C、 $\mathrm{N} 、 \mathrm{P}$ 密度与温度和降水关系显著; $1 \mathrm{~m}$ 深土壤的平均有机C及 $\mathrm{N} 、 \mathrm{P}$ 密度分别为64.0 $\mathrm{Mg} \cdot \mathrm{hm}^{-2} 、 7.1 \mathrm{Mg} \cdot \mathrm{hm}^{-2} 、 4.2 \mathrm{Mg} \cdot \mathrm{hm}^{-2}$, 土壤有机C及 $\mathrm{N}$ 密度与温度和降水关系显著。中国北方灌从生态系统的总有机C及 $\mathrm{N} 、$ $\mathrm{P}$ 储量分别为1.7 Pg、164.9 Tg、124.8 Tg。其中生物量C、 N、P储量分别为 $128.4 \mathrm{Tg} 、 3.1 \mathrm{Tg} 、 0.2 \mathrm{Tg}$; 调落物C、 $\mathrm{N} 、 \mathrm{P}$ 储量分 别为8.4 Tg、0.45 Tg、0.027 Tg; 土壤是最大的C、 $\mathrm{N} 、 \mathrm{P}$ 库, $1 \mathrm{~m}$ 深土壤有机C及 $\mathrm{N} 、 \mathrm{P}$ 储量分别为 $1.6 \mathrm{Pg} 、 161.3 \mathrm{Tg} 、 124.6 \mathrm{Tg}$ 。 关键词＼cjkstart碳；氮；磷；储量；密度；灌从; 中国北方

引用格式: 郭炎培, 杨弦, 安尼瓦尔·买买提, 刘鸿雁, 马文红, 于顺利, 唐志尧 (2017). 中国北方温带灌丛生态系统碳、氮、磷储量. 植物生态学报, 41, 14-21. doi: $10.17521 /$ cjpe.2016.0201

\section{Storage of carbon, nitrogen and phosphorus in temperate shrubland ecosystems across Northern China}

GUO Yan-Pei ${ }^{1}$, YANG Xian ${ }^{1}$, MOHHAMOT Anwar ${ }^{2}$, LIU Hong-Yan ${ }^{1}$, MA Wen-Hong ${ }^{3}$, YU Shun-Li ${ }^{4}$, and TANG Zhi-Yao $^{1 *}$

${ }^{1}$ College of Urban and Environmental Science, Peking University, Beijing 100871, China; ${ }^{2}$ Xinjiang Institute of Ecology and Geography, Chinese Academy of Sciences, Ürümqi 830011, China; ${ }^{3}$ College of Life Sciences, Inner Mongolia University, Hohhot 010021, China; and ${ }^{4}$ State Key Laboratory of Vegetation and Environmental Change, Institute of Botany, Chinese Academy of Sciences, Beijing 100093, China

\section{Abstract}

Aims Studying storage of carbon (C), nitrogen (N) and phosphorus (P) in ecosystems is of significance in understanding carbon and nutrient cycling. Previous researches in ecosystem $\mathrm{C}, \mathrm{N}$ and $\mathrm{P}$ storage have biased towards forests and grasslands. Shrubland ecosystems encompass a wide gradient in precipitation and soil conditions, providing a unique opportunity to explore the patterns of ecosystem $\mathrm{C}, \mathrm{N}$ and $\mathrm{P}$ storage in relation to climate and soil properties.

Methods We estimated densities and storage of organic C, N and P of shrubland ecosystems in Northern China based on data from 433 shrubland sites.

Important findings The main results are summarized as follows: the average organic $\mathrm{C}, \mathrm{N}$ and $\mathrm{P}$ densities in temperate shrubland ecosystems across Northern China were $69.8 \mathrm{Mg} \cdot \mathrm{hm}^{-2}, 7.3 \mathrm{Mg} \cdot \mathrm{hm}^{-2}$ and $4.2 \mathrm{Mg} \cdot \mathrm{hm}^{-2}$, respectively. The average plant C, $\mathrm{N}$ and P densities were $5.1 \mathrm{Mg} \cdot \mathrm{hm}^{-2}, 11.5 \times 10^{-2} \mathrm{Mg} \cdot \mathrm{hm}^{-2}$ and $8.6 \times 10^{-3}$ $\mathrm{Mg} \cdot \mathrm{hm}^{-2}$, respectively, and were significantly correlated with precipitation and soil nutrient concentrations. The average litter C, N and P densities were $1.4 \mathrm{Mg} \cdot \mathrm{hm}^{-2}, 3.8 \times 10^{-2} \mathrm{Mg} \cdot \mathrm{hm}^{-2}, 2.5 \times 10^{-3} \mathrm{Mg} \cdot \mathrm{hm}^{-2}$ and were significantly correlated with temperature and precipitation. The average soil organic $\mathrm{C}, \mathrm{N}$ and $\mathrm{P}$ densities in the top $1 \mathrm{~m}$ were $64.0 \mathrm{Mg} \cdot \mathrm{hm}^{-2}, 7.1 \mathrm{Mg} \cdot \mathrm{hm}^{-2}$ and $4.2 \mathrm{Mg} \cdot \mathrm{hm}^{-2}$, respectively and the former two were significantly correlated with temperature and precipitation. The total organic C, $\mathrm{N}$ and P storage of shrublands in Northern China were 1.7 Pg, 164.9 Tg and 124.8 Tg, respectively. The plant C, $\mathrm{N}$ and P storage were $128.4 \mathrm{Tg}, 3.1 \mathrm{Tg}$ and $0.2 \mathrm{Tg}$,

收稿日期Received: 2016-06-14 接受日期Accepted: 2016-11-10

* 通信作者Author for correspondence (E-mail: zytang@urban.pku.edu.cn) 
respectively. The litter $\mathrm{C}, \mathrm{N}$ and $\mathrm{P}$ storage were $8.4 \mathrm{Tg}, 0.45 \mathrm{Tg}, 0.027 \mathrm{Tg}$, respectively. Soil is the largest C, $\mathrm{N}$ and $\mathrm{P}$ pool in the studied area. The soil organic $\mathrm{C}, \mathrm{N}$ and $\mathrm{P}$ storage in the top 1 meter were $1.6 \mathrm{Pg}, 161.3 \mathrm{Tg}$ and $124.6 \mathrm{Tg}$, respectively.

Key words carbon; nitrogen; phosphorus; storage; density; shrublands; Northern China

Citation: Guo YP, Yang X, Mohhamot A, Liu HY, Ma WH, Yu SL, Tang ZY (2017). Storage of carbon, nitrogen and phosphorus in temperate shrubland ecosystems across Northern China. Chinese Journal of Plant Ecology, 41, 14-21. doi: 10.17521/cjpe.2016.0201

陆地生态系统各组分碳(C)储量的研究有助于 正确评价生态系统在区域及全球C循环中的作用。 近30年来, 我国学者开展了大量估算中国植被C储 量的研究, 特别是在森林(周玉荣等, 2000; Fang et al., 2001; 赵敏和周广胜, 2004; 方精云等, 2007)和 草地生态系统(Ni, 2002; 朴世龙等, 2004; Fan et al., 2008; 方精云等，2010)。作为中国北方重要的植被 类型之一，灌丛在中国陆地生态系统的C循环中具 有重要的作用, 其分布及生长也随全球气候波动而 变化(胡会峰等, 2006)。研究中国北方灌从生态系统 的C储量对全面了解中国陆地生态系统的C储量和C 周转有着重要的意义。然而, 与大量的森林和草地 生态系统C 储量研究相比, 灌丛生态系统 C 储量的 研究很少, 估算结果也存在极大的差异。例如 $\mathrm{Ni}$ (2001)根据不同灌丛生态系统的全球平均植被密度 结合中国灌从分布面积估算得出中国灌从生态系统 $\mathrm{C}$ 密度为 $45.6 \mathrm{Mg} \cdot \mathrm{hm}^{-2}$, 总C储量为 $8.02 \mathrm{Pg}$; 胡会峰 等(2006)搜集中国实测灌丛生物量数据, 用植被平 均 $\mathrm{C}$ 密度法估算得出中国灌从植被 $\mathrm{C}$ 密度为 10.88 $\mathrm{Mg} \cdot \mathrm{hm}^{-2}$, 总 $\mathrm{C}$ 储量为 $1.68 \mathrm{Pg}$, 其中北方地区的总 $\mathrm{C}$ 储量约为 $406.6 \mathrm{Tg}$ 。

作为生态系统养分循环的重要部分, 氮(N)、磷 (P)在生态系统中的储量和分配受到多种因素的影 响(Ellert \& Gregorich, 1996), 也影响到植物生长和 生态系统功能。但与C储量研究相比, 生态系统N、 $\mathrm{P}$ 储量的研究较少。对中国生态系统N、P储量的研 究主要集中于土壤库的估算(Tian et al., 2006; Yang et al., 2007), 对植被N、P库的研究目前还是空白。

本研究采用统一的调查、采样和测试标准, 对 中国北方的典型灌丛生态系统进行了系统的实地调 查, 以此为基础准确估算了中国北方灌丛生态系统 的C、N、P密度和储量, 试图回答如下科学问题: 中 国北方灌从生态系统的C、N、P密度有多大? 环境 如何影响中国北方灌丛生态系统的 C、N、P密度? 中国北方灌从生态系统的C、N、P储量有多大?

\section{1 材料和方法}

\section{1 调查和采样}

在北京、天津、河北、山西、内蒙古、辽宁、 吉林、黑龙江、山东、河南、陕西、甘肃、宁夏、 新疆, 选取 433 个常见灌从样地开展生物量及土壤 调查及采样。在每个样地设置 3 个 $5 \mathrm{~m} \times 5 \mathrm{~m}$ 的样方, 记录样方的各种环境因子和群落概况, 并通过收获 或相关生长方程获取各样方生物量。同时, 对样方 中出现的每种灌木分器官(根、茎、叶), 对草本植物 分地上和地下部分, 测量或估算其生物量并采样 (详细方法及生物量获取方式见Yang等(2014))。样品 经处理后，用于测定其C、N、P含量。

在样地附近挖一个 $1 \mathrm{~m}$ 深的土壤剖面(土壤深度 不足 $1 \mathrm{~m}$ 的样地, 挖至土壤母质), 将剖面按照深度 划分为如下层次: 0-10、10-20、20-30、30-50、50-70 和70-100 cm, 在每层左右侧壁和正面用容积为 100 $\mathrm{cm}^{3}$ 的环刀取样以测定土壤容重与砾石含量。同时, 在每个样方沿对角线用土钻分别分层采集土样 3 钻 用于测定土壤有机C以及 $N$ 、P含量。

\section{2 植物和土壤样品测定}

土钻样品风干后挑出根和砾石, 过100目篎, 植 物样品烘干后粉碎, 调落物样品风干后挑出杂质并 粉碎, 用于C、 $\mathrm{N} 、 \mathrm{P}$ 元素的分析。

样品的全 $\mathrm{C}$ 和全 $\mathrm{N}$ 含量用 $\mathrm{C} / \mathrm{N}$ 元素分析仪(2400 II CHNS/O; PerkinElmer, Boston, USA)测定。为获取 土壤有机 C含量, 利用土壤无机 C 分析仪(Calcimeter 08.53, Eijkelkamp, Giesbeek, The Netherlands) 测定了同一批土壤样品的土壤无机C含量, 两者之 差即为土壤有机C含量。全P含量用酸溶-钼锑抗比 色法, 用硫酸-高氯酸溶解植物或土壤样品中的P, 再用钼锑抗比色法进行测定(John, 1970)。

土壤环刀样品在 $105^{\circ} \mathrm{C}$ 下烘干并称干质量, 挑 出砾石后测定砾石的质量和体积, 计算每一层土壤 样品的容重和砾石含量。 


\section{3 其他数据来源}

研究中所采用的数据还包括气候与植被分布数 据。由于没有实地监测的气候数据, 研究采用 WorldClim数据库(Hijmans et al., 2005)来获取各样 点的年平均气温和年降水量。灌丛植被分布图来源 于中国科学院遥感与数字地球研究所提供的2010年 中国土地覆被图(张磊等, 2014)。该分类图划分38类 地物, 其中灌从包含了常绿阔叶灌从、落叶阔叶灌 从、常绿针叶灌从以及稀疏灌从(荒漠灌丛) 4种类 型, 地面分辨率为 $30 \mathrm{~m}$ 。由于我们的调查只涵盖了 落叶阔叶灌从和稀疏灌从, 而且其他两类灌从在中 国北方分布面积很小(仅占北方灌从总面积的 $0.4 \%$ ), 所以我们只计算落叶阔叶灌从和稀疏灌丛在中国北 方的密度和储量。

\subsection{C、N、P密度和储量计算}

样地尺度植物各器官 $\mathrm{C} 、 \mathrm{~N} 、 \mathrm{P}$ 储量可以由每种 植物各器官样品C、N和P含量乘以相对应的生物量 计算获得。调落物 $C 、 N 、 P$ 储量由 $C 、 N 、 P$ 含量乘 以相应质量获得。各层土壤有机C、全 $\mathrm{N}$ 和全 $\mathrm{P}$ 含量 乘以对应的土壤容重(扣除砾石)可以计算出该层土 壤有机C、N、P储量, 然后用累加法计算 $1 \mathrm{~m}$ 深土壤 的C、N、P储量。以土壤有机C密度计算为例, 其具 体计算公式为:

$$
S O C D=\sum\left(1-g_{i}\right) \times \rho_{i} \times S O C_{i} \times T_{i}
$$

其中, $S O C D$ 为土壤有机C密度, $g_{i}$ 为第 $i$ 层土壤的砾 石含量, $\rho_{i}$ 为第 $i$ 层土壤的容重, $S O C_{i}$ 为第 $i$ 层土壤的 有机C含量, $T_{i}$ 为第 $i$ 层土壤的厚度。 $1 \mathrm{~m}$ 深土壤 $\mathrm{N} 、 \mathrm{P}$ 密度用相同的方法计算。

由于样方调查为坡面面积, 样地尺度计算所获 得的生物量、调落物以及土壤 $\mathrm{C} 、 \mathrm{~N} 、 \mathrm{P}$ 密度需经坡 度校正。校正方法是用地面投影面积(坡度面积与坡 度 $(\alpha)$ 余弦值 $\cos \alpha$ 的乘积)代替坡面面积。

通过中国土地覆被图(张否等, 2014)获得各省 落叶阔叶灌从、稀疏灌从的分布面积(经坡度校正), 乘以各省两种类型灌丛的 C、N、P密度计算出储量, 各省的C、 N、P储量相加即为中国北方灌从生态系 统的C、N、P储量。

\section{2 结果}

\section{1 中国北方灌丛生态系统C、N、P密度}

中国北方灌从调查样地的平均有机C及 $\mathrm{N} 、 \mathrm{P}$ 密 度分别为(69.8 \pm 3.0$) \mathrm{Mg} \cdot \mathrm{hm}^{-2} 、(7.3 \pm 0.3) \mathrm{Mg} \cdot \mathrm{hm}^{-2}$ 、
(4.2 \pm 0.2$) \mathrm{Mg} \cdot \mathrm{hm}^{-2}$ (平均值 \pm 标准误差)。其中, 生物 量C、 N、P 密度分别为 $(5.1 \pm 0.2) \mathrm{Mg} \cdot \mathrm{hm}^{-2} 、(11.5 \times$ $\left.10^{-2} \pm 0.5 \times 10^{-2}\right) \mathrm{Mg} \cdot \mathrm{hm}^{-2} 、\left(8.6 \times 10^{-3} \pm 0.4 \times 10^{-3}\right)$ $\mathrm{Mg} \cdot \mathrm{hm}^{-2}$; 调落物 $\mathrm{C} 、 \mathrm{~N} 、 \mathrm{P}$ 密度分别为 $(1.4 \pm 0.1)$ $\mathrm{Mg} \cdot \mathrm{hm}^{-2} 、\left(3.8 \times 10^{-2} \pm 0.3 \times 10^{-2}\right) \mathrm{Mg} \cdot \mathrm{hm}^{-2} 、(2.5 \times$ $\left.10^{-3} \pm 0.2 \times 10^{-3}\right) \mathrm{Mg} \cdot \mathrm{hm}^{-2} ; 1 \mathrm{~m}$ 深土壤有机C及 $\mathrm{N} 、 \mathrm{P}$ 密度分别为 $(64.0 \pm 0.3) \mathrm{Mg} \cdot \mathrm{hm}^{-2} 、(7.1 \pm 0.3)$ $\mathrm{Mg} \cdot \mathrm{hm}^{-2} 、(4.2 \pm 0.2) \mathrm{Mg} \cdot \mathrm{hm}^{-2}$ (表1)。

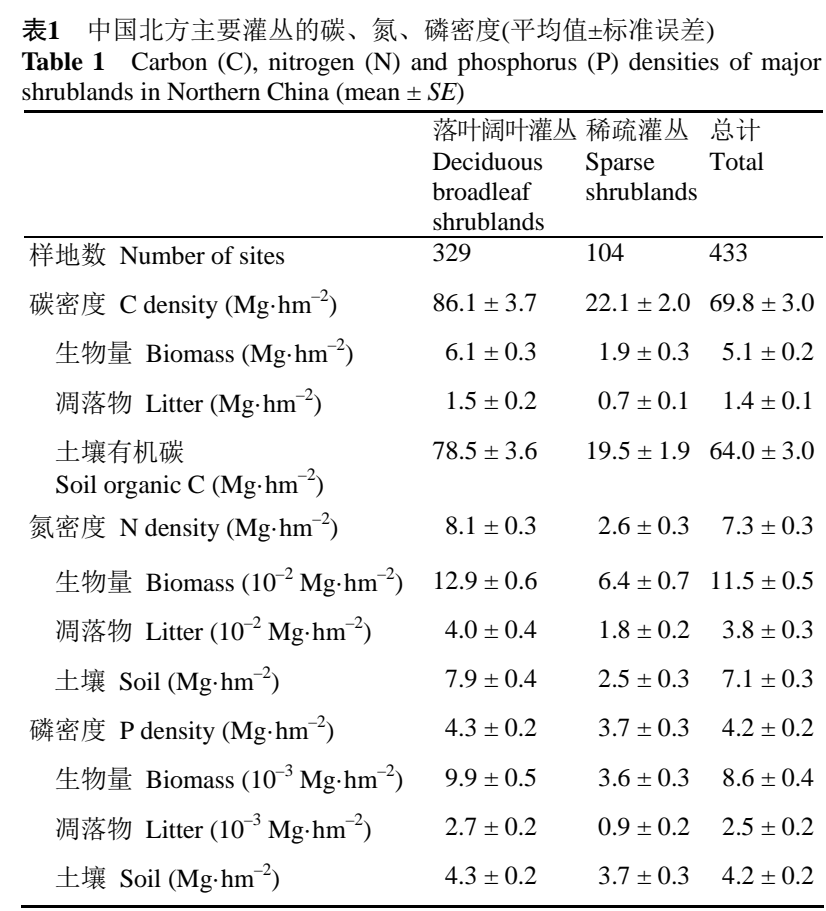

\section{2 中国北方灌丛生态系统C、N、P密度与环境的} 关系

生物量 $\mathrm{C} 、 \mathrm{~N} 、 \mathrm{P}$ 密度与年降水量、土壤养分含 量都显著正相关 $(p<0.001)$, 尤其与年降水量和土 壤 $\mathrm{N}$ 含量的相关度较高。然而它们与年平均气温的 关系相对较弱, 只有 $\mathrm{P}$ 密度与年平均气温相关关系 显著 $(p<0.01)$ (图1)。

调落物 $\mathrm{C} 、 \mathrm{~N} 、 \mathrm{P}$ 密度与年平均气温呈显著的负相 关关系 $(p<0.001)$, 与年降水量有较显著的正相关关 系(对于调落物C、 $\mathrm{N}, p<0.01$, 对于 $\mathrm{P}, p<0.001$ )(图2)。

$1 \mathrm{~m}$ 深土壤有机C与 $\mathrm{N} 、 \mathrm{P}$ 密度随年平均气温升高 显著降低 $(p<0.001)$, 而且土壤有机 $\mathrm{C}$ 与 $\mathrm{N}$ 密度随年 降水量增加显著增加 $(p<0.001)$, 而 $\mathrm{P}$ 密度的变化趋 势较弱(图3)。

\section{3 中国北方灌丛生态系统C、N、P储量}

研究区域灌从总面积为 $2.89 \times 10^{7} \mathrm{hm}^{2}$, 约占 

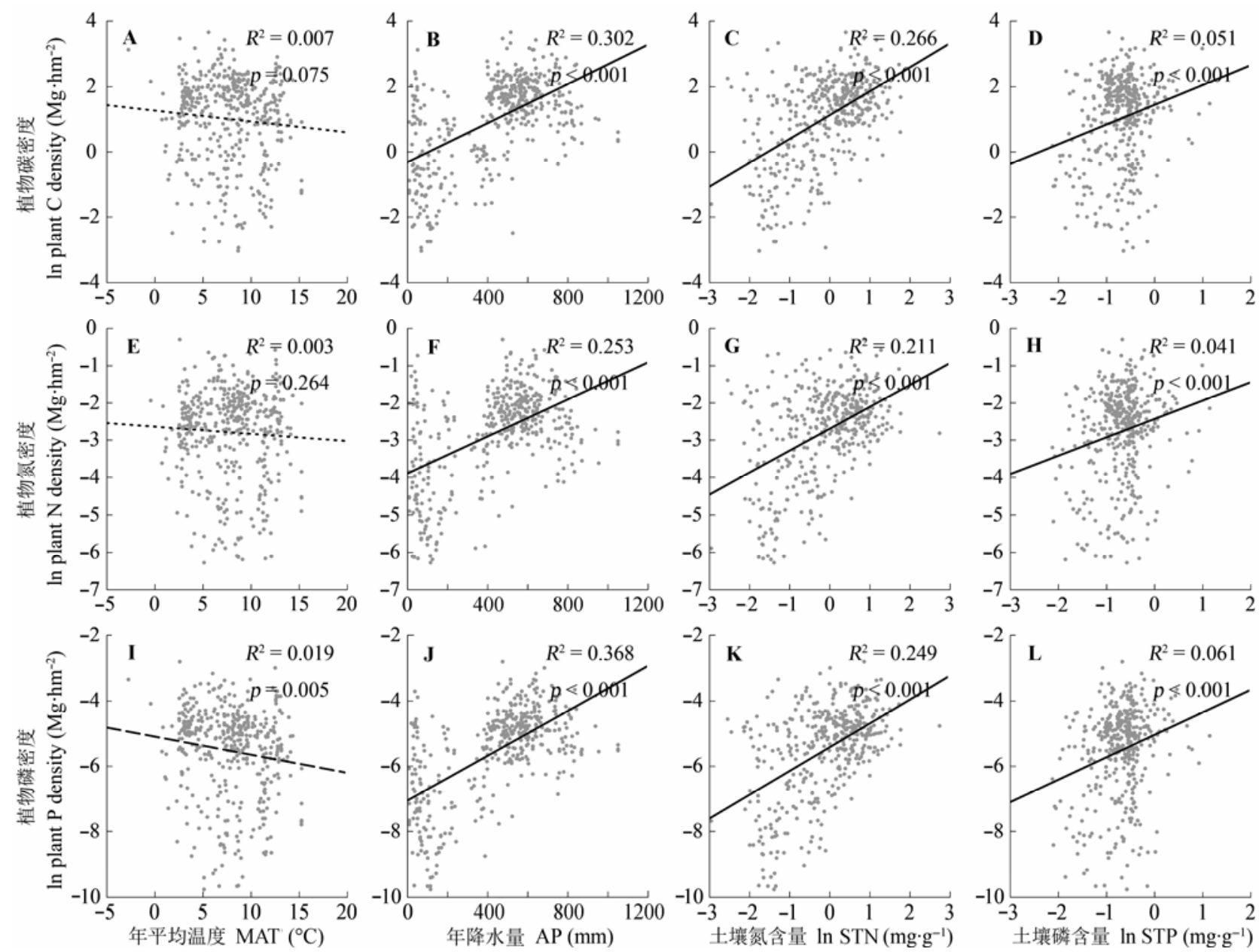

图1 中国北方灌从生物量碳(A-D)、氮(E-H)、磷(I-L)密度与环境的关系(点线: $p>0.01$; 短划线: $0.001<p<0.01 ;$ 实线: $p<$ 0.001)。

Fig. 1 Biomass carbon (C) (A-D), nitrogen (N) (E-H) and phosphorus (P) (I-L) densities for shrublands of Northern China in relation to environmental factors (dotted line: $p>0.01$; dashed line: $0.001<p<0.01$; solid line: $p<0.001$ ). AP, annual precipitation; MAT, mean annual temperature; STN, soil total nitrogen concentration; STP, soil total phosphorus concentration.

研究区陆地总面积的 $5.7 \%$, 其中落叶阔叶灌从和稀 疏灌丛的总面积为 $2.88 \times 10^{7} \mathrm{hm}^{2}$ 。总有机C及 $\mathrm{N} 、 \mathrm{P}$ 储量分别为1.7 Pg、164.9 Tg、124.8 Tg。其中生物 量C、N、P储量分别为 $128.4 \mathrm{Tg} 、 3.1 \mathrm{Tg} 、 0.2 \mathrm{Tg}$; 调 落物 $C 、 N 、 P$ 储量分别为 $8.4 \mathrm{Tg} 、 0.45 \mathrm{Tg} 、 0.027 \mathrm{Tg}$; $1 \mathrm{~m}$ 深土壤有机C与 $\mathrm{N} 、 \mathrm{P}$ 储量分别为1.6 Pg、161.3

$\operatorname{Tg} 、 124.6 \operatorname{Tg}($ 表2)。

\section{3 讨论}

\section{1 中国北方灌丛生态系统C、N、P的密度和储量} 本研究采用统一的调查、采样和测试方法, 计 算了中国北方灌从生态系统C、N、P储量分别为 1.7 $\mathrm{Pg} 、 164.9 \mathrm{Tg} 、 124.8 \mathrm{Tg}$ 。其中生物量C、N、P储量 分别为 $128.4 \mathrm{Tg} 、 3.1 \mathrm{Tg} 、 0.2 \mathrm{Tg}$ 。与以往通过资料
整合以及采用统一的生物量-元素含量转换率进行 的研究相比, 避免了不同采样方式带来的误差, 同 时考虑了不同物种和不同区域植物元素含量的差 异, 可以得到更准确的估算结果。

胡会峰等(2006)估算了中国主要灌从类型的C 密度, 其中温带、亚热带落叶灌从、矮林的 $C$ 密度为 (6.24 \pm 0.88$) \mathrm{Mg} \cdot \mathrm{hm}^{-2}$, 与本研究计算出的落叶阔叶 灌丛C密度 $6.1 \mathrm{Mg} \cdot \mathrm{hm}^{-2}$ 相当。但胡会峰等(2006)估算 的中国灌从植被总 $C$ 密度为 $10.88 \mathrm{Mg} \cdot \mathrm{hm}^{-2}$, 高于本 研究的5.1 Mg. hm ${ }^{-2}$ 。这一方面是因为本研究不包括 生物量较高的常绿灌从, 另一方面是因为本研究额外 包含了生物量较低的稀疏灌从。胡会峰等(2006)估算 的在北方地区灌从生态系统的植被 $\mathrm{C}$ 储量约为 406.6 $\mathrm{Tg}$, 高于本研究结果。这一差异主要来自于对北 

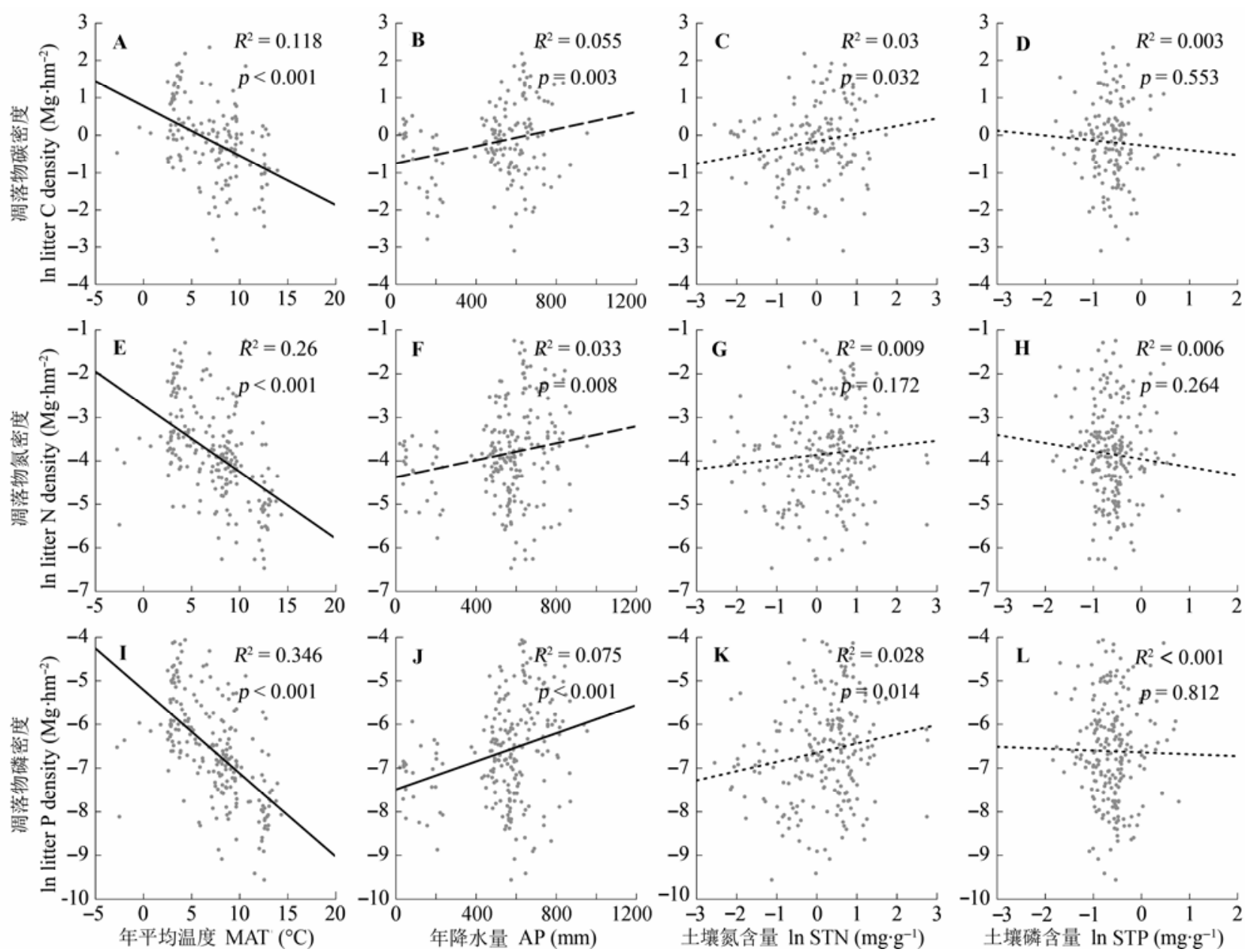

图2 中国北方灌从调落物碳 $(\mathbf{A}-\mathbf{D}) 、$ 氮 $(\mathbf{E}-\mathbf{H})$ 、磷 $(\mathbf{I}-\mathbf{L})$ 密度与环境的关系。不同线型和缩写的意义见图1。

Fig. 2 Litter carbon (C) (A-D), nitrogen (N) (E-H) and phosphorus (P) (I-L) densities for shrublands of Northern China in relation to environmental factors. Please see Fig. 1 for the meaning of different line types and abbreviations.

方灌丛面积的估算, 胡会峰等(2006)采用的植被图 灌从面积比本研究遥感获得的灌从面积大了许多。

中国森林的平均植被 C密度(周玉荣等, 2000; Fang et al., 2001; 赵敏和周广胜, 2004; 方精云等, 2007)是中国北方灌从的平均植被C密度的8倍以上, 这是因为森林生态系统能够承载的生物量密度大大 超过了灌丛生态系统。不同研究对中国草地平均 C 密度的计算结果差异较大 $(\mathrm{Ni}, 2002$; 朴世龙等, 2004; Fan et al., 2008; 方精云等, 2010), 但大体来 看与灌从的植被C密度相差较小(朴世龙等, 2004; 方精云等, 2010)。虽然由于分布面积的差异, 草地 植被 $\mathrm{C}$ 储量大于灌从 $\mathrm{C}$ 储量, 但如果从 C密度角度出 发, 灌从植被 $\mathrm{C}$ 库的重要性并不弱于草地植被C库。

在中国北方灌从生态系统中, 土壤是最大的有 机C、 $\mathrm{N} 、 \mathrm{P}$ 库。1 m深土壤有机C及 $\mathrm{N} 、 \mathrm{P}$ 储量分别为 $1.6 \mathrm{Pg} 、 161.3 \mathrm{Tg} 、 124.6 \mathrm{Tg}$, 分别占生态系统总C、
$\mathrm{N} 、 \mathrm{P}$ 库的 $94.1 \% 、 97.8 \%$ 和 $99.8 \%$ 。对全球森林生态 系统C库的研究表明, 森林生态系统的C库中有约 2/3的C储存于土壤C库(Dixon et al., 1994), 而对中 国草地生态系统C库的研究表明, 有约 $97 \%$ 的C存在 于土壤C库(方精云等, 2010)。中国北方灌从生态系 统的C库的分配介于森林与草地生态系统之间, 与 草地更为相似。与Tian等(2006)和Yang等(2007)的结 果相比, 中国北方灌从的平均土壤 $\mathrm{N}$ 密度不到全国 平均土壤 $N$ 密度的 $1 / 2$, 说明灌从生态系统的土壤较 为贫瘦。

\section{2 中国北方灌丛生态系统 $C 、 N 、 P$ 密度与环境的 关系}

生物量 $\mathrm{C} 、 \mathrm{~N} 、 \mathrm{P}$ 密度随年降水量和土壤 $\mathrm{N} 、 \mathrm{P}$ 含 量增加显著升高, 其中年降水量的拟合优度最高。 水分是中国北方植被分布和生长的限制因素, 水分 的增加促进植物的生长(Bai et al., 2008), 而C作为 

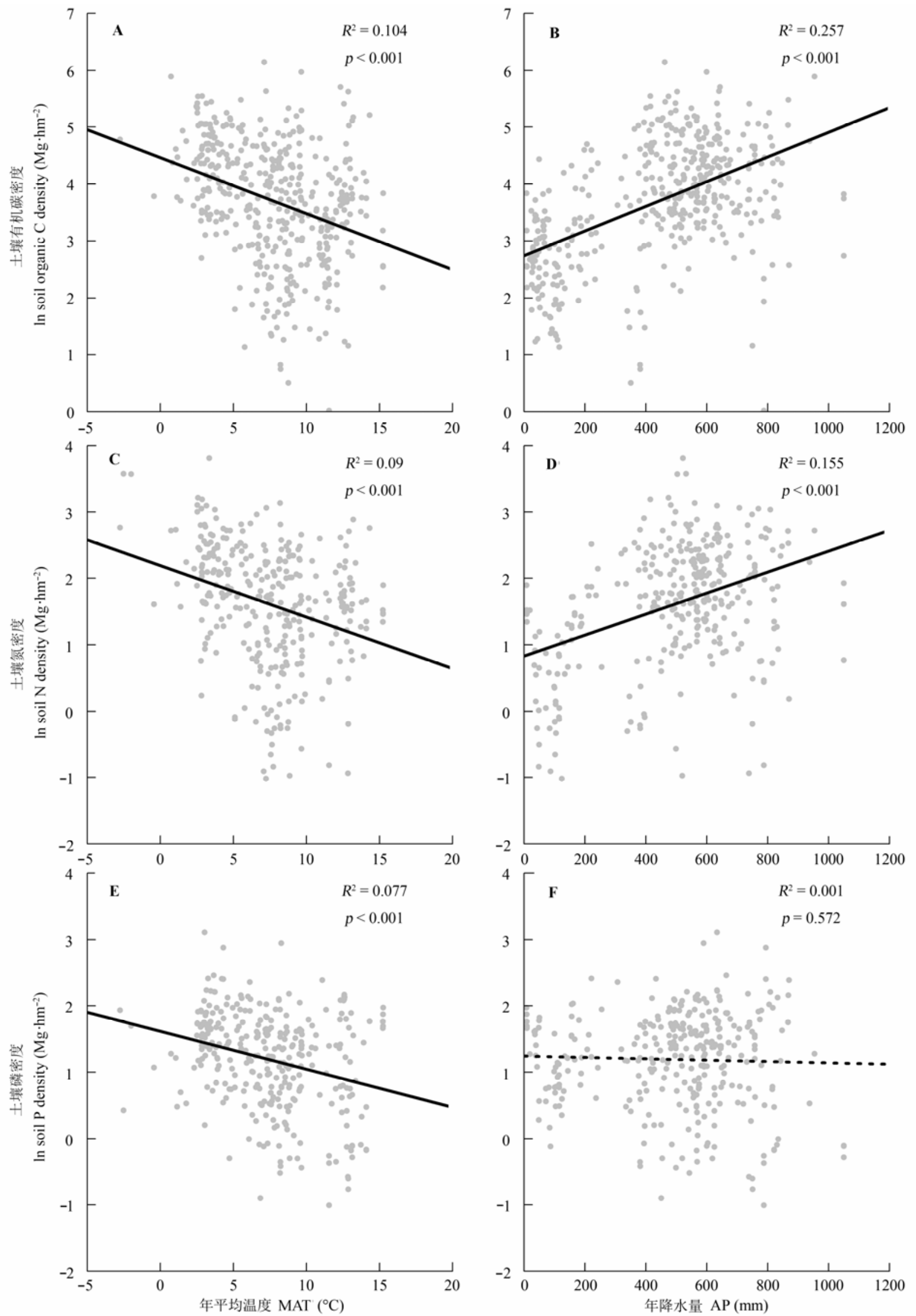

图3 中国北方灌从土壤有机碳 $(\mathbf{A}, \mathbf{B})$ 、氮 $(\mathbf{C}, \mathbf{D})$ 、磷 $(\mathbf{E}, \mathbf{F})$ 密度与气候的关系。不同线型和缩写的意义见图1。

Fig. 3 Soil organic carbon (C) (A, B), nitrogen (N) (C, D) and phosphorus (P) (E, F) densities for shrubland of Northern China in relation to climatic factors. Please see Fig. 1 for the meaning of different line types and abbreviations. 
表2 中国北方主要灌从的碳、氮、磷储量

Table 2 Carbon (C), nitrogen (N) and phosphorus (P) storage of major shrublands in Northern China

\begin{tabular}{lccr}
\hline 灌从植被型 & $\begin{array}{c}\text { 落叶阔叶灌丛 } \\
\text { Deciduous broad- } \\
\text { leaf shrublands }\end{array}$ & $\begin{array}{c}\text { 稀疏灌从 } \\
\text { Sparse } \\
\text { shrublands }\end{array}$ & $\begin{array}{c}\text { 总计 } \\
\text { Total }\end{array}$ \\
\hline 面积 Area $\left(10^{4} \mathrm{hm}^{2}\right)$ & 1828.6 & 1050.4 & 2879.0 \\
碳储量 C storage (Tg) & 1537.6 & 183.5 & 1721.1 \\
生物量 Biomass & 118.6 & 9.8 & 128.4 \\
调落物 Litter & 8.2 & 0.2 & 8.4 \\
土壤有机碳 Soil organic C & 1410.8 & 173.5 & 1584.3 \\
氮储量 N storage (Tg) & 137.1 & 27.8 & 164.9 \\
生物量 Biomass & 2.8 & 0.3 & 3.1 \\
调落物 Litter & 4.5 & 0.0 & 4.5 \\
土壤 Soil & 133.8 & 27.5 & 161.3 \\
磷储量 P storage (Tg) & 80.7 & 44.1 & 124.8 \\
生物量 Biomass & 0.2 & 0.0 & 0.2 \\
淍落物 Litter & 2.7 & 0.0 & 2.7 \\
土壤 Soil & 80.5 & 44.1 & 124.6 \\
\hline
\end{tabular}

储量由各省区不同类型灌从面积与该省区相应类型灌从 $\mathrm{C} 、 \mathrm{~N} 、 \mathrm{P}$ 平均 密度的乘积加和得到, 因此该数据不简单等同于表 1 的密度与总面积的 乘积。

The element storage of each shrubland type is the sum of the area multiplied by corresponding $\mathrm{C}, \mathrm{N}$ and $\mathrm{P}$ densities in each province, instead of the average element density in Table 1 multiplied by total area.

植物生物量最主要的组成元素, 其密度也会随水分 增加而增加。同理, $\mathrm{N} 、 \mathrm{P}$ 密度也与C密度一样随降水 量增加而增加。除了水分以外, 土壤养分含量的增 加也会促进植物的生长(Chapin III, 1980; Chapin III et al., 1986), 所以生物量C、N、P密度也会相应增 加。而土壤 $\mathrm{N}$ 含量与生物量 $\mathrm{C} 、 \mathrm{~N} 、 \mathrm{P}$ 密度的拟合优 度都比土壤 $\mathrm{P}$ 含量更高, 这可能是由于中国北方灌 从生态系统的降水与土壤 $\mathrm{N}$ 含量有较强的相关性 $(r=0.62)$, 降水可能会带来更多的 $\mathrm{N}$ 输入(N沉降和 调落物)(Brooks, 2003; Zhou et al., 2009)。

与生物量不同, 调落物 $\mathrm{C} 、 \mathrm{~N} 、 \mathrm{P}$ 密度都与温度 负相关, 而与降水量正相关。这可能是因为温度升 高促进了微生物分解作用, 减少凋落物量, 从而减 少调落物C、N、P密度(Hobbie, 1996); 而降水促进 了调落物输入, 增加了调落物的 C、N、P密度(Zhou et al., 2009)。

由于土壤中元素的耦合作用(Cleveland \& Liptzin, 2007), 土壤C、N密度随气候因子的变化相同。 土壤中的 $\mathrm{C} 、 \mathrm{~N}$ 主要存在于土壤有机质中, 以土壤 $\mathrm{C}$ 密度为例, 温度升高促进了土壤有机质的分解, 增 加了土壤C流失(Schimel et al., 1994; Jobbágy \& Jackson, 2000), 所以土壤C密度随着年平均气温升 高而下降。降水增加则促进了植物生长, 加速了有
机质的积累, 提高了土壤C密度(Jobbágy \& Jackson, 2000; Wynn et al., 2006)。同理, 土壤N密度也呈现出 类似的格局(Post et al., 1985; Cleveland \& Liptzin, 2007)。中国北方灌丛生态系统的土壤C、N耦合还 表现在土壤C、N密度的空间分布具有较高的相关性 $(r=0.78)$ 。

\section{3 中国北方灌丛生态系统C、N、P储量估算中的} 不确定性

本研究通过大范围调查我国北方典型灌从样地 中植物和调落物的 $\mathrm{C} 、 \mathrm{~N} 、 \mathrm{P}$ 含量以及 $1 \mathrm{~m}$ 深土壤容重 和有机C、N、P含量, 结合遥感数据, 估算了生态系 统C、N、P储量。与以往研究相比, 本研究的方法 能够得到更加准确的估算结果。但是, 本研究的估 算中仍然具有如下来源的不确定性: (1)一些物种的 标准株或样品采集数量不够, 其相关生长方程或元 素含量是利用近缘物种的调查或测试数据建立的。 （2)对C、 N、P储量的估算是将调查样地的平均C、N、 $\mathrm{P}$ 密度推广到同省份相同类型的植被。上述两个近 似处理环节可能会对C、N、P储量的估算带来一些 误差。补充调查数据将会减小这些误差和不确定性, 从而更加准确地估算C、N、P储量。

基金项目中国科学院战略先导性科技专项 (XDA05050301)。

\section{参考文献}

Bai YF, Wu JG, Xing Q, Pan QM, Huang JH, Yang DL, Han XG (2008). Primary production and rain use efficiency across a precipitation gradient on the Mongolia plateau. Ecology, 89, 2140-2153.

Brooks ML (2003). Effects of increased soil nitrogen on the dominance of alien annual plants in the Mojave Desert. Journal of Applied Ecology, 40, 344-353.

Chapin III FS (1980). The mineral nutrition of wild plants. Annual Review of Ecology and Systematics, 11, 233-260.

Chapin III FS, Vitousek PM, van Cleve K (1986). The nature of nutrient limitation in plant communities. The American Naturalist, 127, 48-58.

Cleveland CC, Liptzin D (2007). C, N, P stoichiometry in soil, is there a 'Redfield ratio' for the microbial biomass? Biogeochemistry, 85, 235-252.

Dixon RK, Solomon AM, Brown S, Houghton RA, Trexier MC, Wisniewski J (1994). Carbon pools and flux of global forest ecosystems. Science, 263, 185-190.

Ellert BH, Gregorich EG (1996). Storage of carbon, nitrogen and phosphorus in cultivated and adjacent forested soils of Ontario. Soil Science, 161, 587-603.

Fan J, Zhong H, Harris W, Yu G, Wang S, Hu Z, Yue Y (2008). 
Carbon storage in the grasslands of China based on field measurements of above- and below-ground biomass. Climatic Change, 86, 375-396.

Fang JY, Chen AP, Peng CH, Zhao SQ, Ci LJ (2001). Changes in forest biomass carbon storage in China between 1949 and 1998. Science, 292, 2320-2322.

Fang JY, Guo ZD, Piao SL, Chen AP (2007). Terrestrial vegetation carbon sinks in China, 1981-2000. Science China Earth Sciences, 37, 804-812. (in Chinese) [方精云, 郭兆迪, 朴世龙, 陈安平 (2007). 1981 2000年中国陆 地植被碳汇的估算. 中国科学: 地球科学, 37, 804-812.]

Fang JY, Yang YH, Ma WH, Mohammat A, Shen HH (2010). Ecosystem carbon stocks and their changes in China's grasslands. Science China Life Sciences, 40, 566-576. (in Chinese) [方精云, 杨元合, 马文红, 安尼瓦尔·买买提, 沈海花 (2010). 中国草地生态系统碳库及其变化. 中国 科学: 生命科学, 40, 566-576.]

Hijmans RJ, Cameron SE, Parra JL, Jones PG, Jarvis A (2005). Very high resolution interpolated climate surfaces for global land areas. International Journal of Climatology, 25, 1965-1978.

Hobbie SE (1996). Temperature and plant species control over litter decomposition in Alaskan Tundra. Ecological Monographs, 66, 503-522.

Hu HF, Wang ZH, Liu GH, Fu BJ (2006). Vegetation carbon storage of major shrublands in China. Journal of Plant Ecology (Chinese Version), 30, 539-544. (in Chinese with English abstract) [胡会峰, 王志恒, 刘国华, 傅伯杰 (2006). 中国主要灌丛植被碳储量. 植物生态学报, 30, 539-544.]

Jobbágy EG, Jackson RB (2000). The vertical distribution of soil organic carbon and its relation to climate and vegetation. Ecological Applications, 10, 423-436.

John MK (1970). Colorimetric determination of phosphorus in soil and plant materials with ascorbic acid. Soil Science, 109, 214-220.

Ni J (2001). Carbon storage in terrestrial ecosystems of China, estimates at different spatial resolutions and their responses to climate change. Climatic Change, 49, 339-358.

Ni J (2002). Carbon storage in grasslands of China. Journal of Arid Environments, 50, 205-218.

Piao SL, Fang JY, He JS, Xiao Y (2004). Spatial distribution of grassland biomass in China. Acta Phytoecologica Sinica, 28, 491-498. (in Chinese with English abstract) [朴世龙, 方精云, 贺金生, 肖玉 (2004). 中国草地植被生物量及
其空间分布格局. 植物生态学报, 28, 491-498.]

Post WM, Pastor J, Zinke PJ, Stangenberger AG (1985). Global patterns of soil nitrogen storage. Nature, 317, 613-616.

Schimel DS, Braswell BH, Holland EA, McKeown R, Ojima DS, Painter TH, Parton WJ, Townsend AR (1994). Climatic, edaphic, and biotic controls over storage and turnover of carbon in soils. Global Biogeochemical Cycles, 8, 279-293.

Tian H, Wang S, Liu J, Pan S, Chen H, Zhang C, Shi X (2006). Patterns of soil nitrogen storage in China. Global Biogeochemical Cycles, 20, GB1001. doi: 10.1029/2005GB002464.

Wynn JG, Bird MI, Vellen L, Grand-Clement E, Carter J, Berry SL (2006). Continental-scale measurement of the soil organic carbon pool with climatic, edaphic, and biotic controls. Global Biogeochemical Cycles, 20, GB1007. doi: 10.1029/2005GB002576.

Yang X, Tang ZY, Ji CJ, Liu HY, Ma WH, Mohhamot A, Shi ZY, Sun W, Wang T, Wu X, Yu SL, Yue M, Zheng CY (2014). Scaling of nitrogen and phosphorus across plant organs in shrubland biomes across Northern China. Scientific Reports, 4, 5488. doi: 10.1038/srep05448.

Yang YH, Ma WH, Mohhamot A, Fang JY (2007). Storage, patterns and controls of soil nitrogen in China. Pedosphere, 17, 776-785.

Zhang L, Wu BF, Li XS, Xing Q (2014). Classification system of China land cover for carbon budget. Acta Ecologica Sinica, 34, 7158-7166. (in Chinese with English abstract) [张磊, 吴炳方, 李晓松, 邢强 (2014). 基于碳收支的中 国土地覆被分类系统. 生态学报, 34, 7158-7166.]

Zhao M, Zhou GS (2004). Carbon storage of forest vegetation and its relationship with climatic factors. Scientia Geographica Sinica, 24, 50-54. (in Chinese with English abstract) [赵敏, 周广胜 (2004). 中国森林生态系统的 植物碳咜量及其影响因子分析. 地理科学, 24, 50-54.]

Zhou XH, Talley M, Luo YQ (2009). Biomass, litter, and soil respiration along a precipitation gradient in southern Great Plains, USA. Ecosystems, 12, 1369-1380.

Zhou YR, Yu ZL, Zhao SD (2000). Carbon storage and budget of major Chinese forest types. Acta Phytoecologica Sinica, 24, 518-522. (in Chinese with English abstract) [周玉荣, 于振良, 赵士洞 (2000). 我国主要森林生态系统碳咜量 和碳平衡. 植物生态学报, 24, 518-522.]

责任编委: 谢宗强 责任编辑: 王 蒇 


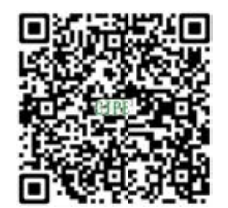

植物生态学报官网

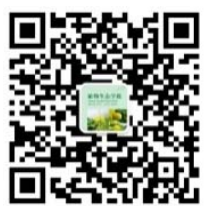

微信订阅号

期刊及学科

相关信息发布

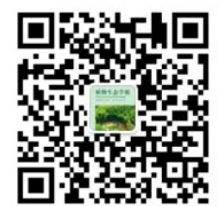

微信服务号

稿件状态查询

全文检索浏览 